\title{
Active libraries: A flexible strategy for active networks
}

David C. Lee and Scott F. Midkiff

Bradley Department of Electrical and Computer Engineering

Virginia Polytechnic Institute and State University

Blacksburg, Virginia, USA 24061-0111

Phone: + 1 (540) 231-2295

Fax: +1 (540) 231-3362

Email:dlee@vt.edu and midkiff@vt.edu

\begin{abstract}
Active networks are a new area of research and as such there are many different ideas as to what an active network should be. One possible architecture is described in this paper along with experimental results that characterize the performance and verify the operation of a fundamental component of the architecture. This component is an active library resolution service that allows active programs to find and load active libraries from the network. This strategy is evaluated and reviewed in detail. The results indicate that the research prototype, which only investigates the issue of where and how to obtain arbitrary libraries from the network, does work and should be scalable. The proposed architecture relies on a novel conceptualization of what minimum functionality should be in an extensible operating system and how systems could be built in an active network. The view is that extensible operating systems and mobile code allow functional components of the operating system and user applications to be obtained from the network. Thus, the overarching goal is a dynamic system in which system modules, such as file systems or network protocols, are loaded into and unloaded from the kernel on demand and the modules are obtained transparently from the network for active programs that need them.
\end{abstract}

Keywords

Active libraries, network protocols, active networks, operating systems

The original version of this chapter was revised: The copyright line was incorrect. This has been corrected. The Erratum to this chapter is available at DOI: 10.1007/978-0-387-35388-3_42 


\section{INTRODUCTION}

The evolution of the modern Internet is a process that has taken decades and has resulted in a robust and flexible service to an ever-growing number of users. This rapid increase in usage and addition of service requirements is straining the Internet and forcing the development of a new protocol for the network, Internet Protocol version 6 (IPv6) (Lee, et al., 1998). As IPv6 development has also taken a number of years and will continue to take a number of years, various "glue" technologies have been and are being used in the interim until there is a pure IPv6 network. The ubiquitous World-Wide Web had been around a number of years before it became popular. These two cases illustrate an important problem in protocol standardization and deployment - it takes a significant amount of time to develop a protocol and to have significant wide-spread adoption of a protocol to make it truly useful. This problem is especially troublesome for protocols that operate at the network or transport layer; users must have a supported platform and/or must be able to get their service providers to support the service. For example, IP multicast service deployment has been resisted by many service providers. One proposed concept for reducing protocol development, deployment, and acceptance time is the active network (DARPA, 1998a and Tennenhouse, et al., 1997). An active network is a network that is dynamically configurable and allows for "rapid injection" of new protocols. This is done by not standardizing on how bits are transferred across the network, but rather on uniform computational models for protocol processing.

This paper presents an architecture that supports the dynamic nature of an active network by allowing active programs to find and load arbitrary active libraries. It discusses the operation and implementation of a research prototype that was used to verify the core component of the architecture, the active library resolution service. The paper also presents the evaluation of this prototype which shows that it works and works well.

\section{ACTIVE NETWORK RESEARCH}

Tennenhouse, et al. (1997), provide an excellent summary of current active network research. Important active network concepts used in this work are briefly reviewed. In the modern network, a packet is the delivery unit that transfers data from one point to another. The concept behind active networks is to make the packet "smarter" by inserting code as data. This code is executed at intermediate switching nodes and allows custom computations to be performed on arbitrary packets. Clearly, there are a number of problems that arise and these are discussed below in relation to the enabling technologies.

A subtle change in how code-carrying packets are viewed can result in a powerful abstraction since, in this case, the packet is not a vehicle for carrying code but it is the code itself. This new vehicle is commonly called a capsule (Tennenhouse and 
Wetherall, 1996). Capsules are self-contained programs that instruct programmable switches on how to process themselves and may leave behind persistent code to help process other capsules.

\section{ENABLING AND RELATED TECHNOLOGIES}

Mobile agents (Nwana, 1996 and IBM, 1997), a type of intelligent agents, are very similar in concept to an active network. Mobile agents are software that can be executed on arbitrary nodes in the network and can forward themselves through the network. The major difference between mobile agents and active networks is that mobile agents are at the application layer and active networks are generally at the network layer.

Key enabling technologies for an active network include extensible operating systems (Engler, et al., 1995, Shapiro, et al., 1997, and Bershad, et al., 1995) and mobile code (Thorn, 1997, Yemini and da Silva, 1996, and Wahbe, et al., 1993). Extensible operating systems take the micro-kernel approach one step further they separate resource management from resource protection (Engler, et al., 1995). Thus, the extensible operating system can dynamically load different modules at run-time, such as memory managers or file systems. A run-time user-customizable kernel has a number of problems such as efficient inter-module communications and secure execution. One clearly does not want a poorly behaved module to terminate the entire operating system.

Mobile code allows platform-independent, or portable, software to be developed. Mobile code technology, such as Java (Gosling, et al., 1996) and NetScript (Yemini and da Silva, 1996), comes in two variants, interpreted and dynamically compiled. Interpreted code is executed by software that performs the function described by the source code or scripting language. An alternative approach to interpretation is to run a machine-neutral bytecode in a virtual machine environment. Dynamically compiled code takes the portable source code and compiles it so that a native machine-code binary can be used. Clearly, dynamic compilation is faster than interpretation; however, there are numerous code-safety issues that must be resolved (Wahbe, et al., 1993 and Keppel, et al., 1991). Both mobile code execution mechanisms should be available in a truly flexible active network environment. The Liquid Software project shows that it is possible to compile simple Java code to native code on a $200-\mathrm{MHz}$ machine in the time it takes to receive the code on a $10 \mathrm{Mbps}$ network connection (Hartman, et al., 1996).

Extensible operating systems can be combined with mobile code to allow truly dynamic operating environments. This combination allows a vendor to write one application that runs on any hardware platform that incorporates the mobile code and extensible operating systems in a standard way. Obviously, much research and standardization remains to be performed in order to efficiently, reliably, and securely perform this task. This research focuses on one extension to, or 
modification of, this model. If the application needs a module, such as a database manager, that is not currently on the system then the application should be able to resolve it from the network. If the application was properly debugged and written, the module that is required must exist somewhere and there is a high probability that it can be found on the network.

\section{ACTIVE NETWORK OPERATING SYSTEMS}

The system model proposed by this work is a synthesis of the active network capsule concept, mobile agent operation, the extensible nature of research operating systems, and use of mobile code. The premise is that there is an operating environment, called the active network operating system (ANOS), that is run-time extensible and supports mobile code. It is divided into three privilege levels, the kernel space, the active handler space, and the user application space, as shown in Figure 1. Operating system "personality modules" are referred to as active handlers. The kernel space only provides basic resource protection and access functions, such as access to a file and prevention of multiple simultaneous writes. The active handler space provides the application services that users require, including file systems, memory managers, network protocol stacks, etc. Thus, different users can select different file systems according to what best suits their need. The minimally required modules are an active code resolution module and a network interface. If the resolution module is robust enough, all other modules can be obtained from the network. The user space is akin to the traditional user space.

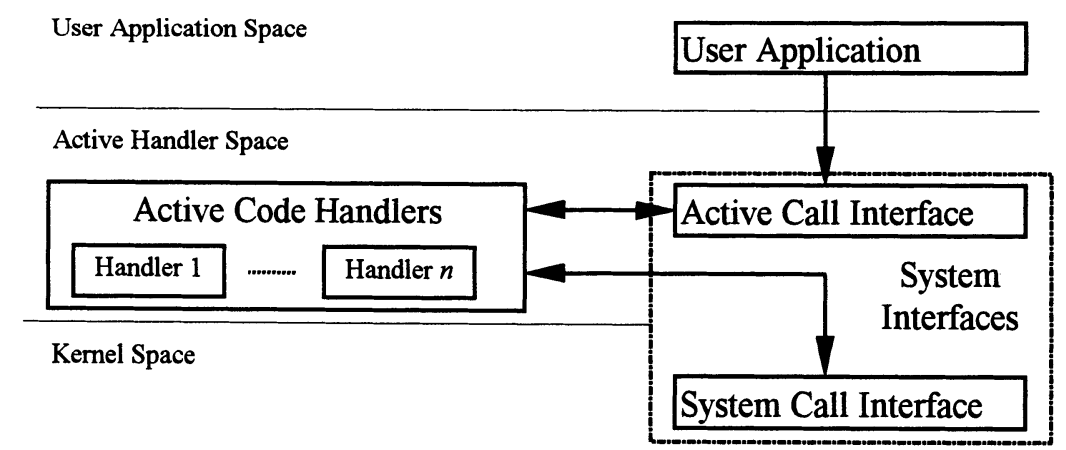

Figure 1 Block diagram of the ideal operating system at a node.

A brief discussion of the active network operating system architecture is given below. Details of the proposed architecture can be found in (Lee, 1998). There are two types of active code, one that exists within the operating system itself and the other that exists in user space. The user space code can be viewed as a mix of traditional applications and mobile agents. The handler code can be viewed as a 
mix of traditional extensible operating system modules and active protocols sent across the network. The discussion focuses on handler code as opposed to user code.

\subsection{System Bootstrap}

Assume that a system is shipped with a minimal operating system of the core handler, a base protocol, and a resolution service. The base protocol provides network services for the resolution service and uses the bare memory protection and access features of the kernel. If a form of Dynamic Host Configuration Protocol (Droms, 1997) is embedded into the resolution service, then administrators at a site can ensure that all nodes are loaded in the same fashion. The only special modification that is required in the proposed resolution service is that a fixed sequence of resolution requests must be used. No other special protocol is required. Upon bootstrap, the node executes the initialization code which performs a set of resolution requests for a compiler, an interpreter, a memory manager, a file system, and so forth. Note that the interpreter and/or compiler must be able to support the native binary format of the machine in question and it may be preferable to include a base dynamic compiler in all active network operating systems. If so, then this compiler should allow itself to be replaced. Once the compiler is installed, all other modules can be made to run on the node in question.

\subsection{Active Handler Resolution}

The precise installation process for active handlers is still an open area of research. There are numerous communications and code safety issues that need to be addressed; however, run-time insertion of dynamic code has been proven to be possible within an Active Bridge (Alexander, et al., 1997a). A model of how the resolution strategy operates from the network standpoint is provided later. The remainder of this section describes how code could be resolved and inserted into the operating system. The assumption is that there is a mechanism to query the network for active code, for the network to return information about the location and properties of active code, and for the network to return the code itself.

Figure 2 shows a block diagram of the resolve handler. Clearly some mechanism must be present to ensure that the code that is received is 1) authorized to be executed by the node, and 2) complete. Assuming that code is somehow received by the system, two levels of authorization checking are performed by the proposed system. The gatekeeper makes a simple high-level check to verify that the code format is supported and the capsule was received intact. The security service provides more detailed authentication mechanisms before the code is either directly loaded into the handler space, compiled into code that can be loaded into the handler space, or interpreted within the handler space. Code that is loaded into the handler space runs using special guard code (Wahbe, et al., 1993) to ensure that 
handler failure does not cause catastrophic failure for the operating system and to ensure that the handler does not try to override operating system protections.

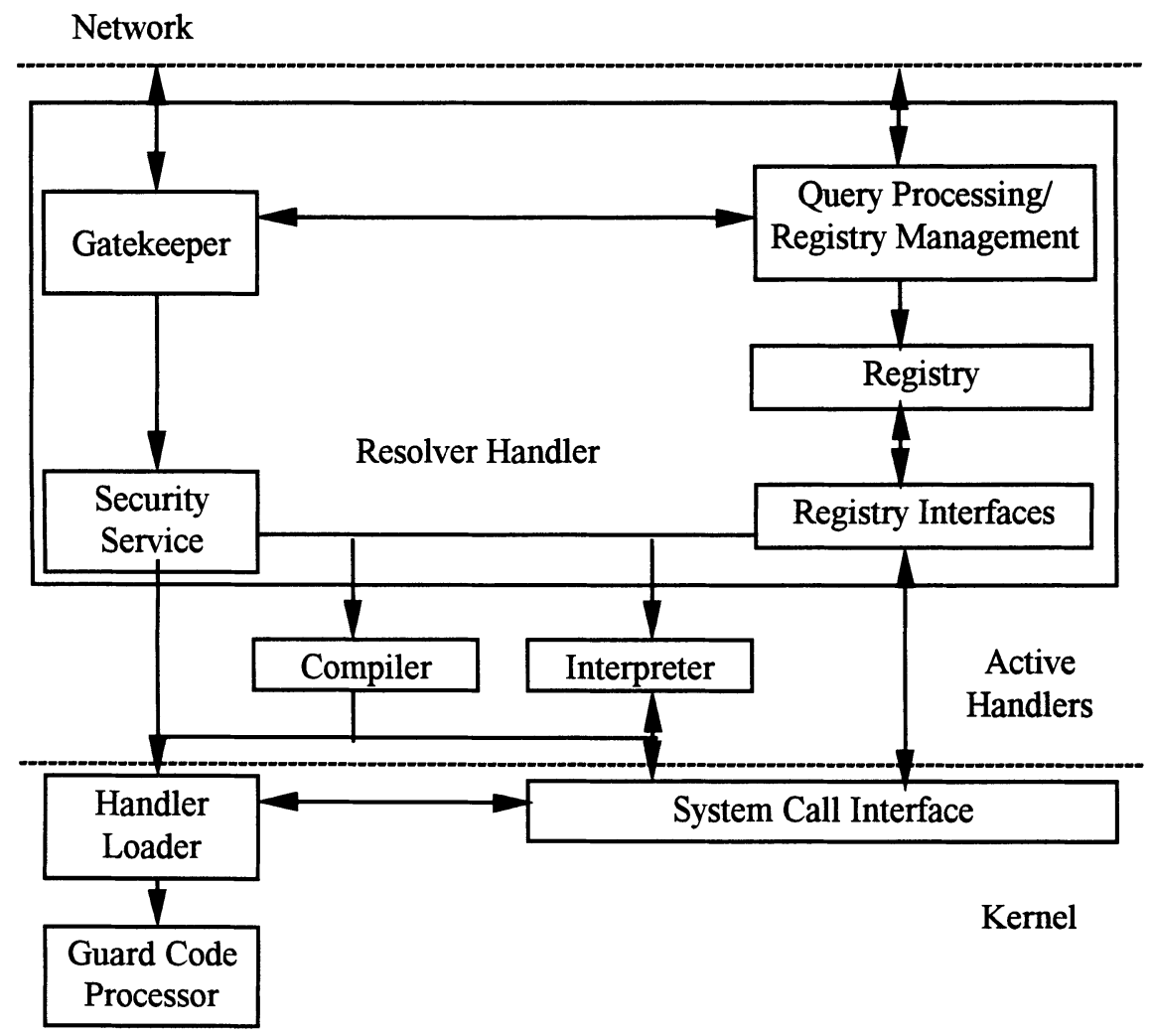

Figure 2 Block diagram of the resolver handler.

Code delivery, from the user space, in the user space, and from the network, may not be complete. For example, a user application may be run that requires a newer windowing system library than is present on the system. Without that library, the application will not run; however, if the code is active in nature then it can make a call to the resolution service which will search the network for the appropriate library. Once that library is found, it can be installed and the application can be run - all without human intervention. Two mechanisms can be used to ensure that the code is complete. The first mechanism is to embed, in special headers transferred along with the code, all the required libraries. The gatekeeper can use this information to verify that required libraries are present and if not present, can either obtain them for the active code or reject the active code. The second mechanism is the on-demand resolution and loading of active libraries. This can be performed by the guard code that is inserted into the active code or by the 
interpreter. The former method allows for efficient, non-stop execution of the active code and the second method ensures that the system is robust.

\section{ACTIVE CODE TRANSPORT AND RESOLUTION}

The remainder of the paper discusses how code can be transported to and from a node and a proposed strategy for resolving libraries from the network. There are two methods that can be used to deliver active code, retrofitting existing protocols or creating a new internet protocol. Clearly, the later is not a preferred solution if the only driving reason to create a new internet protocol is to support active code. Thus, retrofitting IP is the popular solution (Wetherall and Tennenhouse, 1996 and Alexander, et al., 1997b). The solution entails creating a new IP header option, the Active IP option (Wetherall and Tennenhouse, 1996) that can be used to inform a node that the code in the packet is a capsule. Nodes that are aware of the active network can process the code and nodes that are not aware of the active code will simply ignore the code. These retrofitting proposals provide a way to identify the program encoding that is used by the active code and authentication information about the active code. This is insufficient as other information will be required for a robust active network; however, the design of the Active IP option does not preclude this as it uses an encoding similar to Multipurpose Internet Mail Extensions (MIME) headers (Borenstein and Freed, 1996). Thus, if information such as distribution restrictions, copyright and usage cost information, revision history, required libraries, and so forth need to be attached to active code, it can be easily done.

The retrofitting proposals provide a simply delivery mechanism that does not easily allow active code to be retrieved from another node. One can make the argument that a capsule can be sent to the other node which can then retrieve the active code. If so, this would be a standard operation and this capsule can be viewed as part of a standard set of capsules that are available on any active node. The retrieval operation is an important requirement for a robust active library resolution strategy. The active library resolution strategy should be able to request code to be transferred or, upon receipt of a query and finding the library locally, a node should be able to send to code to the requester.

Now that the basic elements of the operating environment and code transfer mechanisms have been developed, the core service of active library resolution is discussed. Active libraries are active code that are used by other active programs. The libraries can be stand-alone programs or other libraries.

Assume the active code arrives at switch $\mathrm{C}$ and passes to switch $\mathrm{B}$ and then to host $A$ and also assume that an active library is required, as shown in Figure 3. Ideally, host A would request the library first from switch $B$ and then from switch $C$ (Tennenhouse and Wetherall, 1996). However, it is possible that switch B and C can either fail or delete required libraries for whatever reason and host A will not 
be able to execute the active code. If the active code is popular, then clearly the active library will be located somewhere on the network; especially considering that users within a group tend to use the same applications (Alexander, et al., 1997a). To handle the situation that the active library may be found along the return path of the code or is available locally, the proposed service relies on the expanding-ring multicast search (Deering, 1991). This mechanism also allows queries to arbitrary and unknown nodes on the network. The expanding-ring multicast search first makes a query to all nodes that are on the same network as the requester, labeled search 1 in Figure 3. If a library is not found on the local network, then the search is repeated so that adjacent networks are included, labeled search 2. Again, if the library is not found, the search range is increased again. If proxy servers are used, then this model can be expanded to handle private networks and potentially provide query translation from different protocols. In addition, caching mechanisms can be used so that special active library servers can be present in the network.

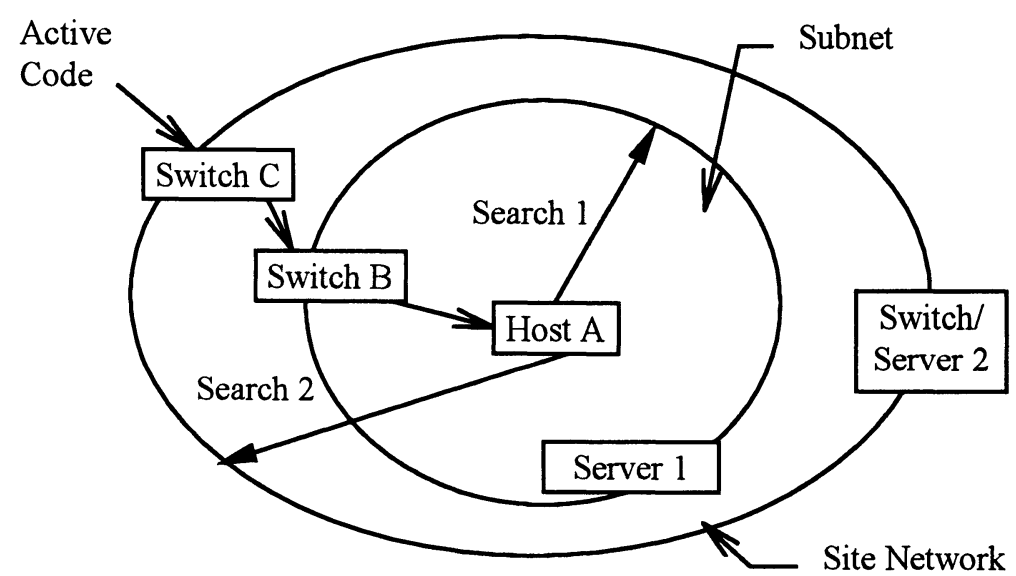

Figure 3 Active library resolution operational model.

\section{PROTOTYPE IMPLEMENTATION AND EVALUATION}

This research developed an experimental protocol to perform active library resolution and a related protocol to transport the active code. The transport protocol was developed to show the features that are required of a transport protocol for an active network. A prototype implementation was created to verify that the resolution and code execution strategy works. As it is impossible to measure the performance of a global service without wide-scale deployment and the later is clearly not possible, a separate simulation system was built to verify that the performance of the system is acceptable and scalable. The simulation 
system was validated against test runs of the small-scale prototype. The remainder of this section discusses key features of the transport protocol and the library resolution protocol and presents the experimental results.

\subsection{Active Code Transport and Resolution}

As discussed earlier, active code transport can be performed by retrofitting existing protocols and should include MIME headers. MIME headers are text-based and are easily extensible. This work developed a test protocol to determine what type of headers are required of an active network and what type of protocol, or capsule, support is needed. A wide range of headers from naming the active code, application programming interfaces (APIs), authoring and copyright information, distribution restrictions, usage cost, and payment were investigated. The proposed use of MIME headers also introduce a dictionary-based compression scheme to reduce the overhead consumed by text headers.

The headers also provide the mechanism to search for a library. Many of the headers can be used as search targets for a query. The searches are performed by making regular expression comparisons and simple numerical tests, as appropriate. If an active program requires an active library, the name of the active code is will typically be used as the search target. Search constraints include verifying that the active code in question has the correct API and, perhaps, version or other information. The search targets and constraints are treated as a logical AND of all previous operations.

Clearly, in a dynamic Internet of the future, code will be treated as a commodity and it is unclear what the economic model will be. Thus, this header investigation must be considered preliminary but the results are that any active code transport mechanism should support active code delivery and retrieval and provide a mechanism to allow capsules at a source node to determine if the remote node properly processed the capsule. As these are common requirements, standard support must be provided. This research implemented a simple protocol called the Active Transport Protocol (ATP) to test these ideas and to provide support for the resolution service.

As noted earlier, the resolution strategy relies on multicast to perform the query. Considering a set of MIME headers are used as search targets and constraints, it is clear that the query will not fit into a single packet. As the API information is expected to be the major component of the search, twenty Linux header files were investigated to determine the likely typical size of an API search.

As shown in Tables 1 and 2, the average function name size is 11 bytes and the average number of parameters per function is three. The average number of composite data types per function is six. This number is obtained by dividing the composite type count of 115 by the 20 header files. The average length of a 
composite data type name is ten bytes and the average number of variables used by a composite data type is four. About 16 functions and 12 composite data types exist in an average header file. Other analyses, reported in (Lee, 1998), confirmed that these numbers are reasonable. Because function parameters typically consist of well-known structures and names, such as the $C$ int or float primitive data types, a high-level of compression can be achieved. Based on this analysis and the typical maximum transfer unit of 1,500 bytes, it is likely that most queries will be one to three packets in length.

Table 1 Header File Analysis Results, Part I

\begin{tabular}{|c|c|c|c|c|c|}
\hline & \multirow[b]{2}{*}{ Count } & \multicolumn{4}{|c|}{ Function Name Size (Bytes) } \\
\hline & & Total & Average & Low & High \\
\hline Function Count & 310 & 3409 & 11.0 & 2 & 30 \\
\hline Composite Types & s 115 & 1065 & 9.3 & 2 & 16 \\
\hline Totals & 425 & 4474 & 10.5 & 2 & 30 \\
\hline
\end{tabular}

Table 2 Header File Analysis Results, Part II

\begin{tabular}{lllll}
\hline & & \multicolumn{3}{l}{ Number of Parameters } \\
& Total (Bytes) & Average & Low & High \\
\hline Function Count & 675 & 2.1 & 0 & 9 \\
Composite Types & 407 & 3.5 & 1 & 26 \\
Totals & 1082 & 2.6 & 0 & 26 \\
\hline
\end{tabular}

Considering the small number of packets required, the Active Library Resolution Protocol (ALRP) was designed to support up to 64 packets to be transmitted, which allows an estimated 590 uncompressed search constraints to be included in 576-byte minimum sized IP packets. If a library requires more than 590 search constraints, it probably is not well written.

ALRP uses no error correction, but relies on the fact that in the expanding-ring search the packets are naturally retransmitted as the ring is expanded. This significantly reduces the complexity of the protocol and the traffic required to transmit error correction information. 


\subsection{Simulation Evaluation}

The prototype system verified that active libraries can be resolved from the network and installed and executed dynamically. A modified ping (Regents, 1993) program was delivered from a source node to a destination node. This ping program required a simple checksum library and this requirement was embedded in the headers. The library was resolved from the network, installed, and the ping program successfully executed.

Table 3 Test Network Characteristics

\begin{tabular}{lllll} 
Case & Group Membership Size & Level & Distance & Number of Leaf Networks \\
\hline 1 & 10 & 0 & 2 & 2 \\
2 & 100 & 0 & 2 & 2 \\
3 & 1000 & 1 & 3 & 10 \\
4 & 10000 & 2 & 5 & 100 \\
5 & 100000 & 4 & 9 & 1000 \\
\hline
\end{tabular}

Since system extensibility can be achieved through the addition of MIME headers, the other primary criteria for a global system is scalability. As noted earlier, the system must be simulated to evaluate performance. The simulator that was created used random hierarchical networks at various fixed levels of hierarchy. Hierarchical networks closely match the topology of a multicast tree (Deering, 1991). A number of different cases were used to show scalability, as indicated in Table 3. The number of nodes in the network and the levels of the network were increased for each case. The system measured the transmission time and number of packets sent and also allowed for different link error rates to be set. Two different end-to-end error rates were calculated and used which were one and five percent. One test case used error rates from one to 75 percent. Because of the lack of error correction, the important factor for scalability was the distance away from the source. Scalability was measured in terms of linear resolution time and packet counts as the distance from requesting client to the designated server increased.

The simulation did not account for the effects of multicast routing, caching, different request sizes and implementations, and similar considerations. The simulation does consider networks of different sizes, different loss rates, variations in server and source locations, and variations in key factors. Bandwidth for all 
links is assumed to be a uniform $10 \mathrm{Mbps}$. Delay and loss rates are assumed to be uniform across all links. The simulator was verified against the prototype for case 1.

\section{Net 4 Loss Analysis, Resolution Time}

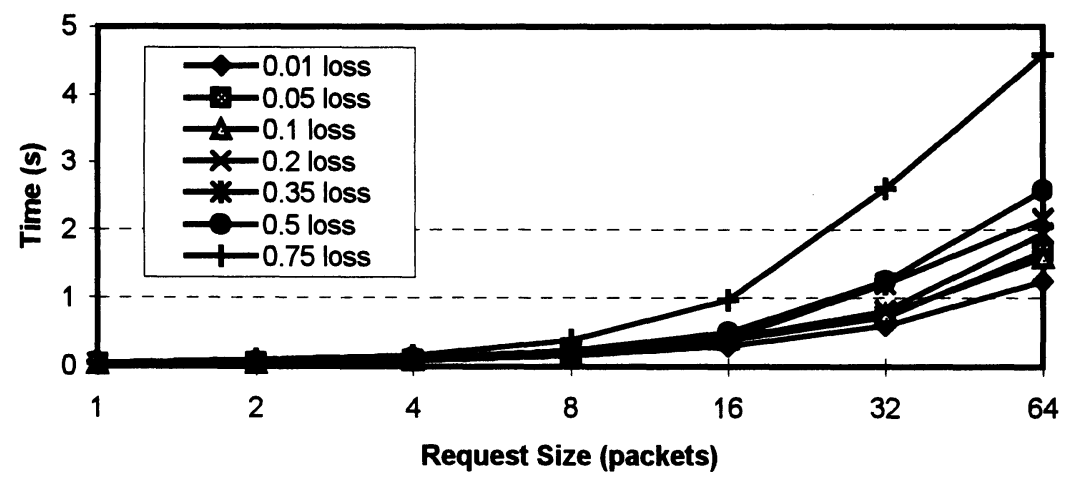

Figure 4 ALRP loss performance analysis for resolution time.

Figure 4 shows the analysis of loss for case 4 . As the request size and the error rate are increased, the resolution time shows a proportional increase. Considering the worst case of 64 packets in a query, there was only a four times increase in the resolution time and a 3.3 times increase in the number of packets transmitted for the 75 percent loss rate compared to the situation with a one percent loss rate. For the typical case of one to three packets, there is a negligible difference in performance.

Figure 5 shows the analysis of scalability for the one percent and five percent error loss situations. This plot gives the resolution time as a function of the number of links and the results presented are an average of the normalized data set. Each data set was normalized to the case where the request size was one packet. The average of the four cases are plotted. For each loss rate, a linear "best-fit" curve is generated and its equation and $R^{2}$ value are presented. The closer the $R^{2}$ value is to unity, the more accurate the linear regression. Figure 5 shows a linear correlation for both the one percent and five percent resolution time cases. The slope for both curves is significantly less than one. Also, both equations are almost the same. This indicates that resolution time scales well, regardless of error rates, and that results for larger networks can be determined by the two similar equations. 


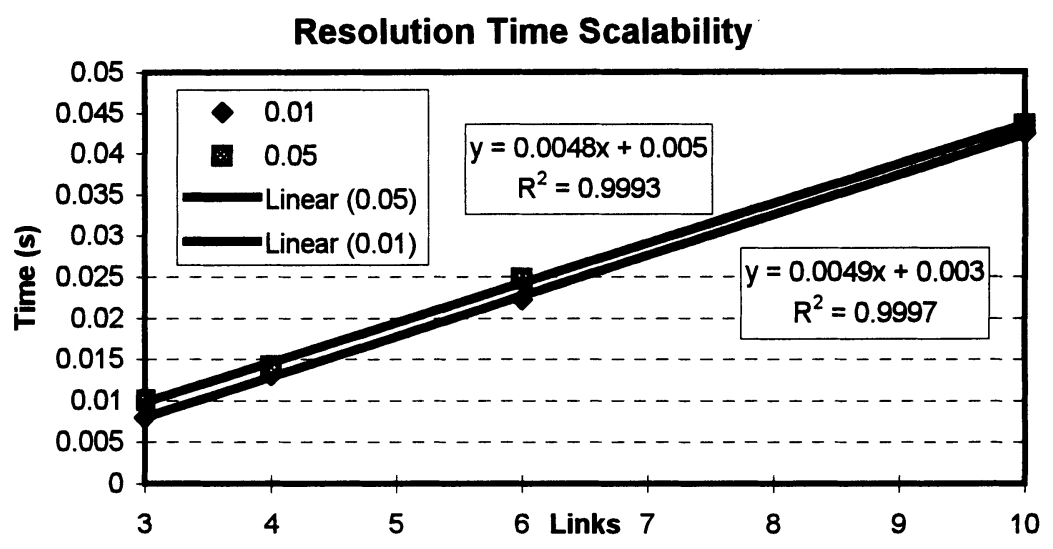

Figure 5 ALRP resolution time scalability.

For transmitted packet counts (not shown), the one percent error case has a slope of 1.3 and the five percent error case has a slope of 19. This indicates that low loss rates scale significantly better than higher loss rates with respect to the transmitted packet counts. Thus, the impact on the network is insignificant for the one percent case and moderate for the five percent case for moderately sized to large networks. From the above analysis, indications are that unrealistically huge networks will have a large number of retransmitted packets at five percent loss. Source to destination distances in modern internets generally are not much more than 15 hops. The significant difference in the two transmitted packet count equations indicates that higher error rates will lead to significantly more packets being retransmitted. Excessive numbers of packets will begin to degrade resolution time performance as network capacity is exceeded. Caching systems should reduce the average distance to something much less than 15 hops.

\section{CONCLUSIONS}

Active networks are an emerging area of research that relies on other emerging areas of research, most notably concepts from extensible operating system and mobile code. This work proposes a unique synthesis of these two areas by advocating a generalized paradigm of dynamic networks and nodal processing. A node consists of a uniform set of kernel interfaces and a service to dynamically obtain other system components from the network. A specific approach to the resolution service was discussed and an evaluation of this approach presented. The use of a flexible transport strategy combined with MIME headers allows for a powerful model of code resolution. Code is treated as an object and the entire network is treated as gigantic database. The scalability of the service is controlled by using expanding-ring multicast searches and caching systems. The 
experimental prototype shows that this approach can work and the simulation results indicate that it can work well on a large scale.

Obviously, there is much research that must be performed before this model, or a variant of it, can be realized. There are significant issues in communications overhead, practical and scalable internal system architectures, standard access models, efficient and safe code execution, and unknown distribution and cost models. This does not even begin to cover the other challenges in active networks (DARPA, 1998b), such as network management and security.

\section{REFERENCES}

Alexander, D.S., Shaw, M., Nettles, S.M., and Smith, J.M. (1997a) Active Bridging. Computer Communication Review, 27:4, 101-111.

Alexander, D.S., Braden, B., Gunter, C.A., Jackson, A.W., Keromytis, A.D., Minden, G.J., and Wetherall, D. (1997b) Active Network Encapsulation Protocol (ANEP). Internet Draft. Work in progress. Available WWW: http://www.cis.upenn.edu/ switchware/ANEP

Bershad, B.N., Savage, S., Pardyak, P., Sirer, E.G., Fiuczynski, M.E., Becker, D., Chamers, C., and Eggers, S. (1995) Extensibility, safety, and performance in the SPIN operating system. Operating Systems Review, 29:5, 267-84.

Borenstein, N. and Freed, N. (1996) MIME (Multipurpose Internet Mail Extensions) Part one: Mechanisms for specifying and describing the format of Internet message bodies. RFC 2045.

Deering, S.E. (1991) Multicast routing in a datagram internetwork. Ph.D. Thesis, Stanford University.

Defense Advanced Research Projects Agency. (1998a) Mission. Available WWW: http://www.darpa.mil/ito/research/anets/index.html

Defense Advanced Research Projects Agency. (1998b) Challenges. Available WWW: http://www.darpa.mil/ito/research/anets/challenges.html

Droms, R. (1997) Dynamic host configuration protocol. RFC 2131.

Engler, D.R., Kaashoek, M.F., and O'Toole, J. (1995) Exokernel: An operating system architecture for application-level resource management. Operating Systems Review, 29:5, 251-266.

Gosling, J., Joy, B., and Steele, G. (1996) The Java language specifications, Version 1.0. Addison-Wesley.

Hartman, J., Manber, U., Peterson, L., and Proebsting, T. (1996) Liquid software: A new paradigm for network systems. Technical Report TR 96-11, University of Arizona. Available FTP: ftp://ftp.cs.arizona.edu/xkernel/Papers/tr96-11.ps

IBM, Inc. (1997) IBM Aglets workbench - home page. Available WWW: http://www.trl.ibm.co.jp/aglets/

Keppel, D., Eggers, S.J., and Henry, R.R. (1991) A case for runtime code generation. Technical Report UW-CSE-91-11-04, Department of Computer Science and Engineering, University of Washington. Available FTP: $\mathrm{ftp}: / / \mathrm{ftp} . c s$. washington.edu/tr/1991/11/UW-CSE-91-11-04.PS.Z 
Lee, D.C., Lough, D.L., Midkiff, S.F. , Davis, IV, N.J., and Benchoff, P.E. (1998) The next generation of the Internet: aspects of the Internet Protocol version 6. IEEE Network, 12:1, 28-33.

Lee, D.C. (1998) Active library resolution in active networks. Ph.D. Thesis, Virginia Polytechnic Institute and State University.

Nwana, H.S. (1996) Software Agents: An Overview. Knowledge Engineering Review, 11:3, 205-244.

The Regents of the University of California (1993) Ping.

Shapiro, J.S., Muir, S.J., Smith, J.M., and Farber, D.J. (1997) Operating system support for active networks. Available WWW: http://www.cis.upenn.edu/ eros/devel/sigcomm97.300dpi.ps

Tennenhouse, D.L. and Wetherall, D.J. (1996) Towards an active network architecture. Computer Communication Review, 26:2, 5-18.

Tennenhouse, D.L., Smith, J.M., Sincoskie, W.D., Wetherall, D.J., and Minden, G.J. (1997) A survey of active network research. IEEE Communications Magazine, 35:1, 80-86.

Thorn, T. (1997) Programming languages for mobile code. ACM Computing Surveys, 29:3, 213-239.

Wahbe, R., Lucco, S., Anderson, T.E., and Graham, S.L. (1993) Efficient software-based fault isolation. Operating Systems Review, 27:5, 203-216.

Wetherall, D.J. and Tennenhouse, D.L. (1996) The ACTIVE IP Option. Proceedings 7th ACM SIGOPS European Workshop, Connemara, Ireland. Available WWW: http://www.tns.lcs.mit.edu/publications/sigops96ws.html

Yemini, Y. and da Silva, S. (1996) Towards programmable networks. Proceedings IFIP/IEEE International Workshop on Distributed Systems. Available WWW: http://www.cs.columbia.edu/ dasilva/content/netscript/pubs/dsom96.ps

\section{BIOGRAPHY}

David C. Lee is a Visiting Assistant Professor in the Bradley Department of Electrical and Computer Engineering at Virginia Tech. He received the B.S. in Computer Engineering and the M.S. and Ph.D. in Electrical Engineering from Virginia Tech. His research interests include advanced network architectures, active networks, and run-time reconfigurable network hardware architectures.

Scott F. Midkiff is an Associate Professor in the Bradley Department of Electrical and Computer Engineering at Virginia Tech. He received the B.S.E. and Ph.D. degrees from Duke University and the M.S. from Stanford University, all in electrical engineering. His research interests include network protocols, wireless networks, and the application of network technology for education. 